\title{
La tradition orale dans le chant grégorien
}

Jacques Viret

\section{OpenEdition}

Journals

Édition électronique

URL : http://journals.openedition.org/ethnomusicologie/2296

ISSN : 2235-7688

Éditeur

ADEM - Ateliers d'ethnomusicologie

Édition imprimée

Date de publication : 1 janvier 1988

Pagination : 46-62

ISBN : 2-8257-0159-9

ISSN : $1662-372 X$

Référence électronique

Jacques Viret, "La tradition orale dans le chant grégorien », Cahiers d'ethnomusicologie [En ligne],

1 | 1988, mis en ligne le 15 août 2011, consulté le 02 mai 2019. URL : http://journals.openedition.org/ ethnomusicologie/2296 


\title{
LA TRADITION ORALE DANS LE CHANT GRÉGORIEN
}

\author{
Jacques Viret
}

Pour l'Occidental moderne la transmission de la musique s'effectue presque exclusivement sur la base de l'écriture - mais il s'agit là d'un fait de culture et de civilisation de portée très générale - , de sorte que le domaine de ce qu'on est convenu d'appeler la «tradition» prend alors, sous le rapport de la musique, l'aspect d'un amas gigantesque d'œuvres, de traités et de commentaires écrits. L'enseignement oral n'a de ce fait plus guère d'autre fonction que d'apprendre à l'élève à se servir correctement des documents écrits dont il dispose et qui renferment jusqu'en ses moindres détails la matière de l'enseignement. De plus, si l'on s'interroge sur le rôle de l'écriture appliquée à la musique, on s'aperçoit que la notion même d' «œuvre» en découle : c'est par l'écriture, et par elle seule, que le musicien peut créer une "œuvre " analogue à un poème, un tableau ou une sculpture, c'est-à-dire un objet esthétique matérialisé sous une forme tangible, autonome et par essence immuable. L'œuvre musicale existe dans la mesure où existe une notation susceptible de la fixer avec une précision suffisante, mais cette notation conserve l'œuvre d'une manière seulement virtuelle tant qu'un exécutant ne vient pas l'actualiser en la traduisant en sons audibles. Importance de la fixation par l'écriture, dichotomie du compositeur qui crée et de l'exécutant qui joue ou chante: ce sont là deux caractéristiques fondamentales de la pratique musicale propre à l'Occident. Caractéristiques à ce point ancrées dans les habitudes qu'elles semblent aller de soi et qu'elles ont fait naître une tendance spontanée à les extrapoler dans les musiques des siècles antérieurs. Que ce soit là une source de contresens plus ou moins graves, on s'en doute, et cela même pour des musiques relativement récentes: rappelons par exemple l'aspect souvent sommaire de la notation musicale baroque, laissant place d'une part à toutes les finesses interprétatives réservées à l'enseignement direct du maître à l'élève (donc à la «tradition orale»), d'autre part à un apport improvisé de l'exécutant, censé assumer vis-à-vis de l'œuvre une fonction partiellement créative (et pas uniquement selon l'acception métaphorique moderne de l'interprète "recréateur»); or l'improvisation représente une pratique typiquement liée au monde de l'oralité, précisément parce qu'elle échappe par définition à l'emprise de l'écrit. On voit donc que la tradition écrite et la tradition orale entretiennent entre elles des relations mouvantes et ambiguës jusque dans les territoires très rationalisés de notre musique classique. 
Il devra par conséquent en aller de même, à bien plus forte raison, pour le vaste corpus de chant liturgique qualifié - légitimement ou non - de "grégorien", considéré à bon droit comme le plus ancien fleuron et comme le véritable soubassement du patrimoine musical d'Occident. Ce répertoire remonte jusqu'aux tout premiers siècles de l'ère chrétienne: il occupe en réalité un millénaire d'histoire ou presque, car jusqu'à l'an mil on ne connaît aucune autre forme de musique que lui (avec aussi quelques répertoires liturgiques - vieux-romain, ambrosien, etc. - qui lui sont parallèles et présentent les mêmes traits stylistiques). Il semble naturel d'assimiler les divers types de chants - antiennes d'introït ou de communion, alléluias, répons, etc. - aux «œuvres» constituant l'héritage musical de l'Occident. On les lit dans le graduel ou l'antiphonaire avec le même œil et dans le même esprit qu'on lira une fugue de Bach ou une sonate de Beethoven, analysant tout à loisir les méandres de la courbe mélodique à l'instar de telle modulation ou de tel développement thématique d'un morceau de l'époque classique. Inconsciemment peut-être on imagine un "compositeur» ayant "mis en musique » un texte sacré et cherché à créer librement une belle mélodie, appropriée à la structure et au sens de ce texte. Or, on le devine, les conditions de l'acte créateur diffèrent radicalement pour une monodie grégorienne et pour une œuvre classique, et ceci en grande partie parce que le répertoire grégorien plonge ses racines dans l'oralité et que les normes régissant la composition et le style grégoriens s'apparentent de près à ce que l'on peut observer, aujourd'hui encore, dans les traditions musicales demeurées vivaces sous cette même forme.

\section{Notations primitives : complémentarité de l'écrit et de l'oral}

Le chant grégorien, tel que nous le connaissons et l'étudions, recèle une sorte de paradoxe, car en lui davantage probablement que partout ailleurs s'interpénètrent traditions écrite et orale. Il ne sera point superflu de rappeler quelques faits à ce sujet, afin de mieux circonscrire le rôle respectif qu'y jouent l'une et l'autre. Commençons par la composante concrète et tangible, celle de la tradition écrite. Dans l'état actuel de la science, l'origine de cette tradition remonte à la fin $\mathrm{du} \mathrm{IX}^{\mathrm{e}}$ siècle, date présumée du plus ancien graduel (recueil des chants de la messe) répertorié sous le $\mathrm{N}^{\circ} 359$ de la Bibliothèque conventuelle de Saint-Gall et suivi par le manuscrit 239 de la Bibliothèque municipale de Laon ${ }^{1}$; un autre manuscrit saint-gallois, le $\mathrm{N}^{\circ} 390-91$ copié par le moine Hartker, est le premier en date des antiphonaires (recueil des chants de l'office monastique) ${ }^{2}$. Cependant, d'après J. Smits van Waesberghe $(1957: 213)^{3}$, on trouverait des traces de notations musicales dès le tout début du IX ${ }^{\mathrm{e}}$ siècle. Des environs de l'an 800 également datent des graduels non notés (textes seuls), intéressants surtout pour l'histoire de la liturgie ${ }^{4}$. C'est donc bien dans le courant du $\mathrm{IX}^{\mathrm{e}}$ siècle que paraissent se situer les débuts de la notation musicale des chants liturgiques. Peut-on sur ce point avoir une certitude absolue? Il serait exagéré de le prétendre, et d'aucuns n'ont pas manqué de faire remarquer que le haut degré d'élaboration et de complexité 
de certaines de ces notations (Saint-Gall et Laon, les plus anciennes précisément) est difficilement envisageable comme une genèse et postulerait donc l'existence hypothétique de livres notés qui ne nous seraient point parvenus et constitueraient les prototypes de ceux que nous possédons ${ }^{5}$. Il semble néanmoins qu'il faille s'en tenir, pour la diffusion généralisée de la notation neumatique, à la date du milieu du $\mathrm{IX}^{\mathrm{e}}$ siècle, établie par des indices solides ${ }^{6}$. Ces anciens témoins notés du chant grégorien, comme beaucoup d'autres jusqu'au XIV et même au XV $\mathrm{XV}^{\mathrm{e}}$ siècle, ignorent encore la portée et transcrivent la mélodie au moyen d'un système de signes analogues à ceux de la sténographie, appelés pour cette raison in campo aperto ("en terrain découvert»), ou encore «neumes purs» ou «neumes-accents» (en considérant leur formation probable à partir des accents grammaticaux). La portée, quant à elle, apparaît progressivement à des dates variables selon les endroits, dès le $\mathrm{XI}^{\mathrm{e}}$ siècle, de pair avec la "diastématie" consistant à visualiser l'acuité et la gravité des notes mélodiques par leur position plus haut ou plus bas sur le parchemin.

Faut-il regarder l'invention de la portée comme un perfectionnement, ainsi que le suggérerait un examen superficiel du processus? D'une certaine manière oui (nous y reviendrons), mais non si l'on tient compte des répercussions néfastes que cette invention a entraînées. Nous voilà au cœur du problème, puisque le système de notation adopté à telle époque et en tel lieu reflète manifestement la conception particulière de la "tradition" du chant liturgique ayant cours à cette époque et en ce lieu. Ce système se révèle donc solidaire des modalités propres à la transmission du chant envisagée globalement, de sorte qu'il est apte à fournir de précieuses indications sur ces modalités elles-mêmes, notamment sur la complémentarité des deux aspects de la transmission, l'écrit et l'oral. Or, si nous nous rapportons à l'époque des plus anciens manuscrits notés, à savoir le $\mathrm{IX}^{\mathrm{e}}$ siècle, nous constatons qu'il existait non pas un mais $d e u x^{7}$ systèmes de notation distincts: il ne faut pas oublier en effet le système alphabétique dont le principe est hérité de l'Antiquité à travers Boèce qui l'a adapté à l'alphabet latin. Ce système avait l'avantage, comparativement à l'écriture neumatique sans portée, de consigner la hauteur exacte des notes et des intervalles; mais il n'était guère usité que pour la théorie, et le seul manuscrit où on le rencontre - le $\mathrm{H} 159$ de la Faculté de Médecine de Montpellier, $\mathrm{XI}^{\mathrm{e}}$ siècle ${ }^{8}$ - servait à l'étude plutôt qu'à la pratique au chœur, vu que les chants y sont groupés par modes et non selon le cursus liturgique comme à l'ordinaire. Si donc l'on s'abstenait d'utiliser une notation qui était certainement connue et qui eût pu indiquer avec précision la hauteur des sons, rendant ainsi quasi superflue la transmission orale, c'est justement que l'on ne voulait point se passer de l'enseignement direct, «de vive voix» (viva voce), peut-être parce qu'on l'estimait inhérent à la qualité même de la transmission" ${ }^{9}$, conformément aux habitudes très généralement répandues dans toute civilisation traditionnelle pour ce qui touche d'une manière ou d'une autre à la doctrine sacrée. La notation neumatique servait alors simplement d'aide-mémoire: elle n'eût été d'aucune utilité à ceux qui n'auraient pas appris les mélodies préalablement (comme un puits si l'on n'a pas de seau, aimait-on à dire). Sous l'angle mélodique, elle indique 

focen domine bedeficiom eft enum uobir $f \perp f^{1}$

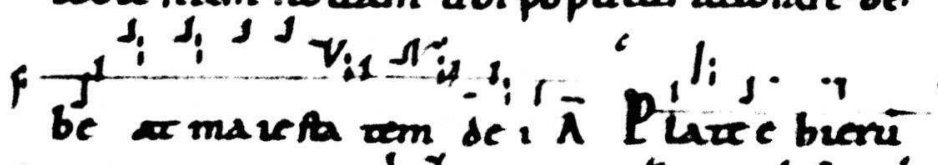

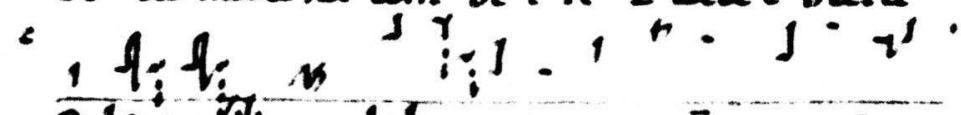
ralem a Jigaudebure ex par orrer uncore.

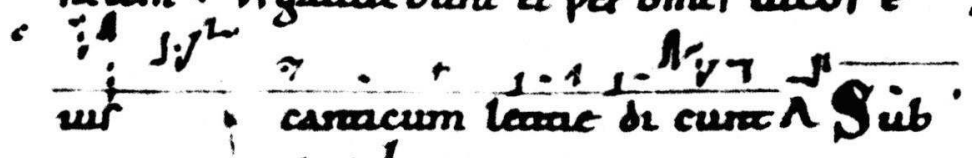

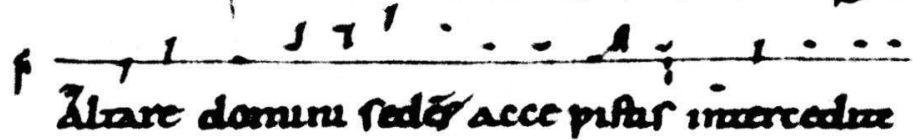

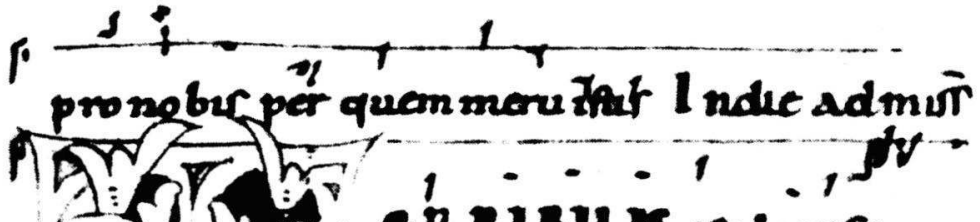

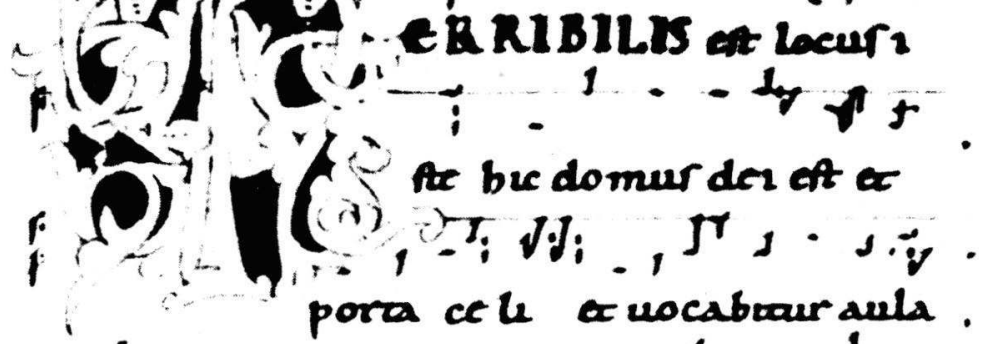

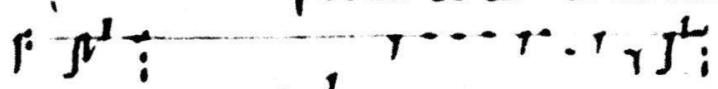
de $2 \overline{P S}$ Pundamena v 6 lonora L ocur $\therefore$

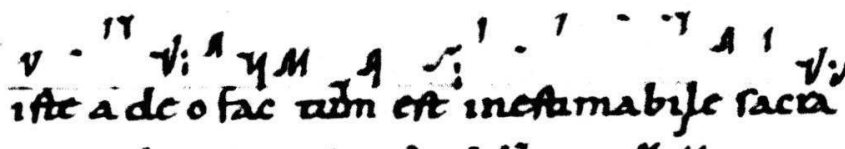
$\Sigma$

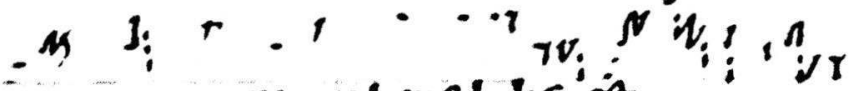
men wam in reprehenribiler ift 


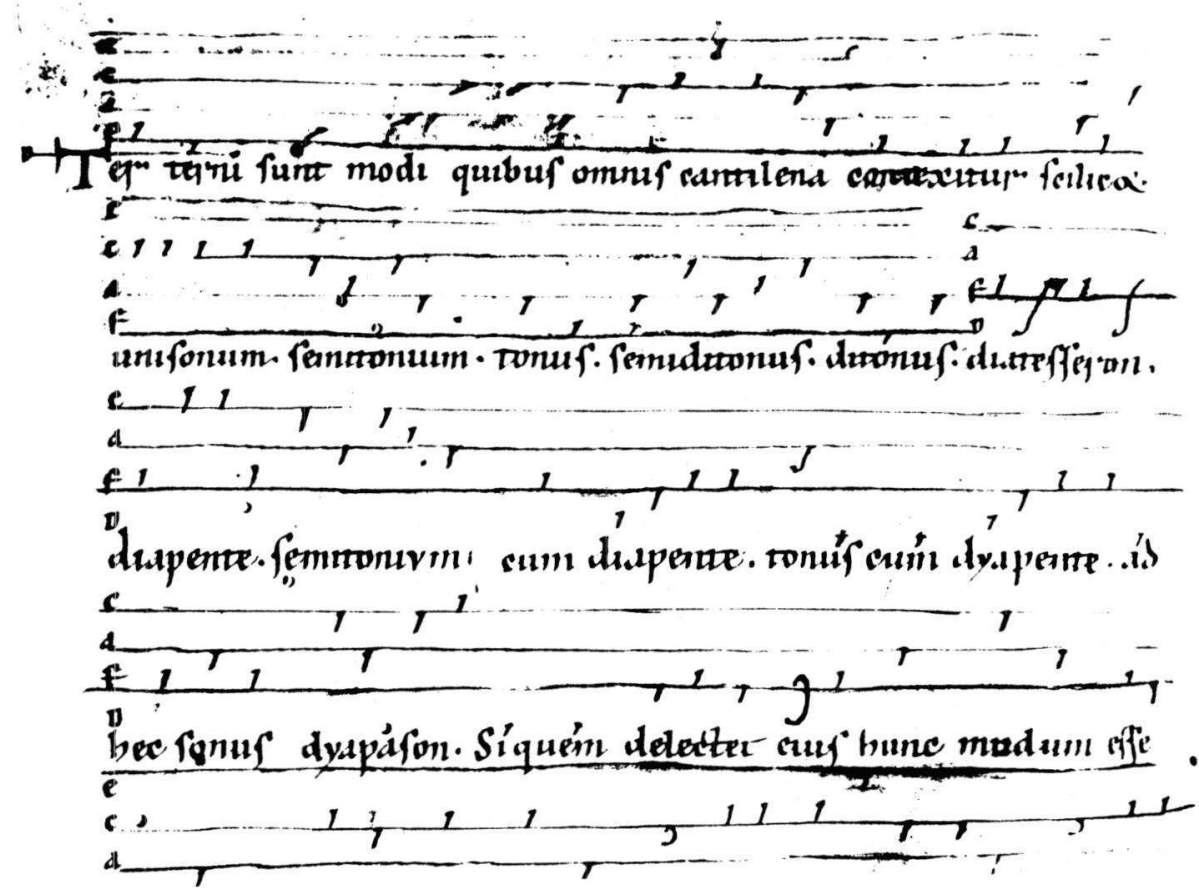

Mélodie didactique sur les intervalles musicaux, d'après un manuscrit du XII ${ }^{\mathrm{e}}$ siècle : portée de quatre lignes marquées des clefs $f$ a $c$ e (fa la do mi) ou df a c (ré fa la do) (Rochester, Sibley Musical Library, Acc. 149 667).

seulement la direction ascendante ou descendante de la ligne entre deux notes successives, mais sous l'angle rythmique elle se révèle - en ce qui concerne surtout les séméiographies de Saint-Gall et Laon - d'une méticuleuse précision. Hors de toute théorie proportionnelle rationnellement codifiée, à l'instar du "mensuralisme» instauré au XIV" siècle et sur lequel repose la "mesure» moderne, la notation neumatique détermine la "lourdeur» ou la "légèreté» de chaque note mélodique, soit en modifiant le tracé graphique des signes, soit par l'adjonction de signes supplémentaires ou de «lettres significatives ». Une formule courante telle que les quatre notes $f a-s o l-f a$ - ré pourra ainsi se noter de cinq façons différentes au moins, correspondant à autant d'articulations rythmiques distinctes. Il est plausible, comme on l'a avancé, que la forme des neumes reproduise le geste du maître de chœur, qu'elle soit donc une "chironomie» : on décrira par exemple un geste arrondi pour la succession de deux notes légères et un geste anguleux pour celle de deux notes lourdes, et c'est aussi de cette façon que sont différenciées par la notation les deux variantes neumatiques correspondantes. 


\section{L'an mil : une étape cruciale de mutations}

L'introduction de la portée, aux alentours de l'an mil, représente bien plus qu'une simple évolution ou qu'un perfectionnement de la notation neumatique in campo aperto. Elle instaure véritablement une nouvelle attitude face à la transmission même du répertoire liturgique, en allégeant considérablement l'effort de mémorisation: désormais tous les intervalles mélodiques seront indiqués avec exactitude, et non seulement leur direction ascendante ou descendante. Le mérite de cette innovation revient à un pédagogue ingénieux - davantage que grand théoricien -, Guy d'Arezzo, qui vécut durant la première moitié du $\mathrm{XI}^{\mathrm{e}}$ siècle. S'il n'a pas, comme on le prétend parfois, inventé le principe même de la portée, du moins en a-t-il tiré tout le parti possible en vue de cet allégement du travail de mémorisation qui semble avoir été son but. Dans sa lettre au moine Michel $^{10}$ il se vante d'avoir, grâce à ce procédé, réduit le temps d'apprentissage de dix à deux années! Jusqu'alors, en effet, un long et pénible labeur - astreignant tant pour le maître que pour l'élève - était requis par la transmission viva voce des chants de la messe (au nombre de plusieurs centaines) aux garçons des scholae cantorum, prototype des futures "maîtrises". Chaque chant, l'un après l'autre, devait être seriné par le maître jusqu'à ce que l'élève soit capable de le reproduire sans faute: discipline fastidieuse qu'un musicologue moderne compare au gavage d'une oie $^{11} \ldots$ Fastidieuse certes, mais sans doute payante si l'on considère la distance qu'il y a entre la lettre "qui tue» et l'esprit "qui vivifie», distance qui n'est autre en réalité que celle des deux aspects de la tradition. D'un point de vue utilitaire et pratique, l'invention de Guy d'Arezzo constitue sans conteste un progrès décisif: mais ce point de vue est essentiellement matériel, donc partiel. Nous avons insisté plus haut sur la préoccupation rythmique particulière dont témoignent les plus anciennes séméiographies; or cette préoccupation disparaîtra dans la mesure exacte des progrès de la notation nouvelle sur portée, comme si l'on perdait sur un plan, celui du rythme, ce que l'on gagne sur l'autre, celui de la mélodie. C'est pourquoi, lorsqu'on veut de nos jours fournir aux choristes un texte musical complet et précis, on est obligé de superposer les deux types d'écriture, celle de Saint-Gall ou Laon en neumes purs pour l'articulation rythmique, et celle sur portée adoptée dès les premières éditions «restaurées" de Solesmes (selon des modèles du $\mathrm{XIII}^{\mathrm{e}}$ siècle) pour les intervalles mélodiques. Après le Graduel neumé de Dom Eugène Cardine, paru en 1966, le Graduale Triplex ${ }^{12}$, la plus récente édition du graduel qui est une version complétée du précédent, répond opportunément à un tel besoin. Certaines tentatives de synthétiser en une notation unique les données rythmiques et mélodiques ont été réalisées depuis peu ${ }^{13}$ : ce serait évidemment, si l'on y parvenait avec succès, la solution idéale parce que la plus claire pour l'œil.

C'est donc aux alentours de l'an mil que commence, selon toute vraisemblance, cette fameuse "décadence» qui s'aggravera régulièrement et durera jusqu'à la «restauration» entreprise à l'abbaye de Solesmes, vers 1850, par Dom Prosper Guéranger et son équipe de moines. Décadence qui s'est opérée en deux phases successives: jusqu'à l'époque de la Renaissance seule l'inter- 

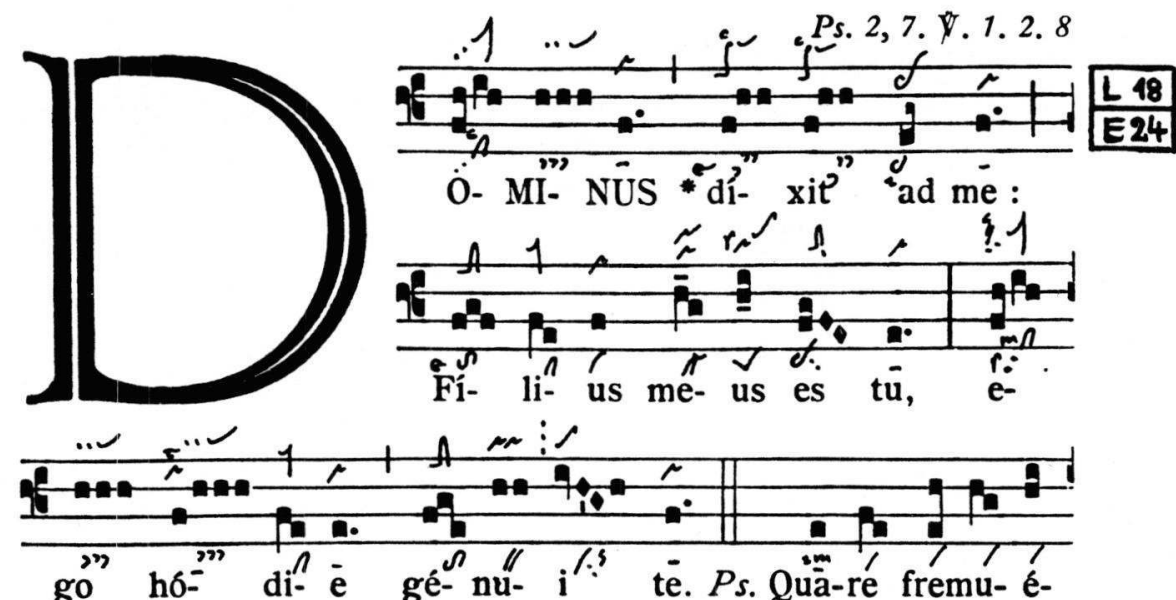

Fí- li- us me- us es tú, e-

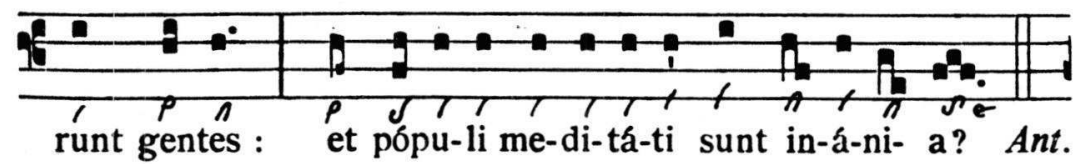

L'introït de la Messe de Minuit selon le Graduale Triplex (Solesmes 1979) : la notation carrée sur portée (d'après les modèles des XIII ${ }^{\mathrm{e}}-\mathrm{XIV}{ }^{\mathrm{e}}$ siècles) est complétée par les neumes-accents de Laon ( $\mathrm{X}^{\mathrm{e}}$ siècle) en bas et par ceux d'Einsiedeln (école de Saint-Gall, $\mathrm{XI}^{\mathrm{e}}$ siècle) en haut.

prétation fut affectée, dans le sens d'un alourdissement et d'une perte des subtilités rythmiques originelles. Puis vinrent les remaniements de la mélodie même, en des éditions semblables à la fameuse "Médicéenne" de 1614, indice plus tangible de l'incompréhension foncière des siècles classiques à l'égard du style et de l'esthétique grégoriens. La transmission du répertoire fut alors dénaturée d'une manière telle qu'on peut se demander s'il existait encore à ce moment quelque chose de vivant et d'authentique de ce même répertoire, élaboré un millénaire plus tôt, ou si au contraire le corpus grégorien n'était pas réduit à l'état de squelette.

Il vaut la peine de se pencher sur les causes et les symptômes de cette décadence pour éclairer le problème plus général de la transmission d'un répertoire, largement assurée originellement par voie orale et vouée à une mainmise croissante de l'écrit. N'y aurait-il point, logiquement, une relation entre ces deux faits, décadence d'une part, changement de type de transmission de l'autre? Mais où est la cause, et où la conséquence? Est-ce parce que la notation se transforme que la pratique d'exécution s'alourdit, ou viceversa l'adoption d'un nouveau type de notation ne serait-il point la manifestation concomitante d'une nouvelle mentalité qui s'exprimerait également par d'autres faits? Dans cette dernière hypothèse Guy d'Arezzo serait le 
représentant de cette nouvelle mentalité, et le caractère très pragmatique de ses diverses inventions pédagogiques (Viret 1985) - la plus importante, à part la diffusion de la portée, étant celle de la solmisation hexacordale, fondement de notre solfège - nous le présente effectivement sous un angle éminemment «moderne», propre à conforter une telle supposition. Il n'est sûrement pas fortuit par ailleurs que la mutation en cause soit contemporaine d'un autre fait lourd de conséquence, à savoir la généralisation et le développement du chant polyphone appliqué au même répertoire liturgique (apparition du "déchant» par mouvement contraire et de l'" organum à vocalises", dans le courant du $\mathrm{XI}^{\mathrm{e}}$ siècle). Ici comme là on a affaire à deux indices complémentaires d'un certain rationalisme orientant en Occident la conception même de la musique vers des voies neuves. Et dans l'ordre de la transmission du répertoire grégorien l'évolution de la polyphonie n'a probablement pas été sans effet: nous pouvons à bon droit admettre qu'elle a contribué, au même titre que le changement de notation et l'importance croissante de l'écrit au détriment de la transmission orale, à l'alourdissement du style d'exécution, premier symptôme de décadence. De langage actuel et pleinement vivant qu'elle était jusqu'à l'an mil, la monodie liturgique en viendra à être vue et traitée comme un legs du passé, et cette nouvelle optique se traduira conjointement par l'intérêt de plus en plus prépondérant porté à la technique polyphonique et par l'altération rythmique de l'exécution. Une question vient à l'esprit à ce propos : est-ce que parce que la notation sur portée était inférieure aux neumes purs dans la capacité de transcrire l'articulation rythmique de la mélodie qu'elle a provoqué une transformation de l'exécution, ou au rebours est-ce parce qu'on se souciait moins de subtilités rythmiques qu'on n'a plus ressenti, à partir d'un certain moment, la nécessité de les noter? Là encore, où est la cause et où la conséquence? Est-on même bien sûr que ces subtilités se soient perdues dès l'instant où la notation ne les a plus indiquées avec exactitude? Il faut certes se garder de tout schématisme et ne point en déduire que le style d'exécution s'est modifié du jour au lendemain (d'autant plus que, répétons-le, les neumes purs se sont maintenus en certains endroits jusqu'à la fin du Moyen Age); néanmoins, l'expression cantus planus que l'on rencontre à partir de 1250 est bien représentative de cette cantilène "plane", c'est-à-dire unie, en notes égales, qu'était devenue la monodie liturgique.

Quant à savoir pourquoi l'aspect rythmique de la mélodie s'est vu le premier atteint, c'est là un point particulièrement intéressant à approfondir. Gardons-nous d'exagérer l'importance de l'écriture dans des processus évolutifs tels que celui-ci: l'écriture n'est après tout que la trace matérielle d'une activité intellectuelle qu'elle reflète, et si elle se transforme, ce ne peut être que parce qu'un changement plus profond s'opère dans cette activité même. Or nous avons vu que le développement de la polyphonie, technique en soi étrangère aux procédés de notation, constituait le signe indubitable d'un pareil changement, et cela prouve bien que les artifices de notation ne sont pas seuls en cause. La frontière chronologique du premier et du second millénaire de l'ère chrétienne correspond assez exactement au point de 
partage entre les deux conceptions fondamentales de la monodie et de la polyphonie, celle-ci représentant la direction spécifiquement moderne et occidentale alors que celle-là apparaît quant à elle reliée au passé et à l'Orient. Il est donc compréhensible que l'engagement progressif dans les voies nouvelles de la polyphonie aille de pair avec une désaffection tout aussi progressive visà-vis de la tradition antérieure, celle de la monodie. C'est de cela précisément, selon nous, que témoignent corrélativement l'adoption d'un nouveau type de notation pour le répertoire monodique, l'alourdissement rythmique de l'exécution, l'emprise croissante de l'écrit et la désuétude de la transmission orale. Et si la désaffection en cause se manifeste en premier lieu dans l'ordre rythmique, cela tient au fait qu'en lui réside l'aspect le plus intérieur, le plus spirituel de la mélodie, donc le plus rebelle à toute rationalisation et, par conséquent, à toute explicitation par l'écriture. Il ne faut pas se leurrer sur la minutie rythmique, que nous avons soulignée, des notations en neumes purs: ces notations, non plus qu'aucune autre notation, ne sont réellement capables de consigner dans sa globalité le mouvement dynamique du rythme vécu et senti, autrement dit l'essence même du rythme musical. La hauteur des notes est, au contraire, un élément aisément objectivable, mesurable et donc transcriptible et transmissible: voilà pourquoi la notation s'est restreinte plus ou moins à cet aspect matériel. Voilà aussi pourquoi l'élément rythmique, vu son caractère subtil et spirituel, subit plus que tout autre et avant tout autre le contrecoup de la mutation rationalisatrice liée au développement de la polyphonie. En théorie, la tradition orale eût pu préserver le style grégorien dans sa pureté et son authenticité, nonobstant l'imprécision rythmique de la notation, mais ce n'est point ainsi qu'il en alla en fait, ce qui confirme que l'affaiblissement de la tradition orale et le renforcement proportionnel de la tradition écrite sont bien dus à une transformation profonde de la mentalité régnante et à cette désaffection (d'abord certes très relative) à l'égard de la monodie que nous avons signalée. Le passage de l'oral à l'écrit s'accompagne d'un inévitable amoindrissement qualitatif dont témoigne la perte du rythme vivant que même une séméiographie aussi précise que celles de Saint-Gall et Laon était incapable de noter intégralement (les nuances d'intensité en particulier en étaient absentes). Si la tradition orale a pu éventuellement suppléer pendant un temps aux lacunes rythmiques de la notation sur portée, cela n'aura été qu'un palliatif éphémère: de même à l'époque baroque la transmission des subtilités interprétatives échappant à la notation n'excèdera point une ou deux générations (on se plaindra par exemple, à l'époque de Rameau, que l'interprétation des opéras de Lully ne soit plus conforme à ce qu'elle était trente ou cinquante ans auparavant). Ainsi, lorsque la transmission d'un répertoire se fonde sur l'écriture et que l'on attribue à cette dernière un rôle plus important que celui d'un simple aide-mémoire, la fidelité de la transmission en pâtit dans la mesure même de cette importance. C'est la porte ouverte à l'individualisme et à la subjectivité, aux «interprétations » conçues au sens moderne du terme, c'est-à-dire comme la vision personnelle que donne un interprète d'un texte écrit, en fonction de la marge d'indétermination nécessairement contenue dans le schéma graphique. 


\section{Aux sources de la tradition grégorienne : la pure oralité}

Mais revenons aux plus anciens manuscrits pourvus d'une notation musicale, lesquels situent, on l'a vu, à la fin du $\mathrm{IX}^{\mathrm{e}}$ siècle le commencement de la tradition grégorienne écrite. Nous avons jusqu'à présent envisagé la transmission du répertoire en aval de cette frontière chronologique, alors que se concurrençaient les deux types de tradition, orale et écrite, avec prédominance rapide de la seconde. Il y a lieu maintenant de reculer en amont du $\mathrm{IX}^{\mathrm{e}}$ siècle, période où la transmission était vraisemblablement assurée par le seul canal oral, tout au moins en ce qui concerne la composante musicale du chant, qui seule nous intéresse ici. On est alors amené à soulever l'épineux problème des origines, puisque celui de la transmission en découle directement. Qu'est-ce au juste que le répertoire grégorien ? Quand, où et comment s'est-il constitué ? Pendant combien de temps sa transmission orale a-t-elle duré ? Quelles altérations éventuelles a-t-il pu alors subir? Autant de questions fort délicates dont la réponse ne repose que sur de maigres indices et témoignages, propres à susciter des hypothèses et non des certitudes. Hypothèses souvent contradictoires jusque sur les points essentiels, et sources de discussions et de polémiques entre spécialistes, sur lesquelles il n'est ni possible ni nécessaire de s'étendre ici. Bornons-nous à indiquer l'alternative posée par l'état du répertoire dont témoigne l'apparition de la tradition écrite aux $\mathrm{IX}^{\mathrm{e}}-\mathrm{X}^{\mathrm{e}}$ siècles : soit cette tradition orale est antérieure, et les manuscrits notés permettraient alors de remonter à un passé plus reculé ; soit ces manuscrits ne valent que pour l'époque dont ils émanent et non pour une période plus ancienne, ce qui équivaut ou bien à nier la fidélité de la tradition orale, ou bien à postuler l'intervention de certaines circonstances historiques particulières ayant entraîné diverses modifications du répertoire. Alternative qu'il serait bon au surplus de nuancer, en considérant le caractère composite de beaucoup de manuscrits où coexistent des «stratifications» multiples qu'il importe au premier chef de discerner: quelle qu'elle soit, écrite ou orale, la "tradition" n'est jamais un bloc homogène mais bien un ensemble complexe d'éléments de provenance et d'ancienneté variables, avec emprunts et influences réciproques d'une école à l'autre. En tout état de cause, c'est à la première solution qu'il faut se rallier si l'on veut maintenir la conception traditionnelle d'un chant "grégorien", c'est-à-dire attribué sinon quant à sa composition - ce que plus personne aujourd'hui n'admet - du moins quant à sa refonte ou à sa codification au pape saint Grégoire le Grand qui occupa le trône pontifical de 590 à 604, et en associant évidemment à cet illustre pontife les musiciens ou chantres ayant oeuvré sous son égide. Conception à laquelle on a objecté de nombreux faits et que bat en brèche tout spécialement la "thèse franque", formulée et abondamment discutée il y a une trentaine d'années: selon cette thèse, ce que nous appelons "grégorien" serait en réalité le remaniement opéré dans la seconde moitié du $\mathrm{VIII}^{\mathrm{e}}$ siècle par les chantres francs du royaume de Pépin le Bref, sur la base d'un répertoire adventice dénommé par convention "vieux-romain » et qui serait alors le seul authentique "chant romain" (Viret 1986: chap. IV). Entre autres difficultés soulevées par une telle hypothèse figure en bonne place l'invraisemblance 
d'un semblable remaniement en l'absence de notation musicale; peut-on raisonnablement l'imaginer abstraction faite de tout support écrit et dans les conditions de la seule tradition orale?

Aucune objection décisive, croyons-nous, ne s'oppose au maintien de la thèse traditionnelle, celle de l'origine romaine et - au moins indirectement "grégorienne", ce qui implique la fidélité d'une transmission orale s'étendant sur trois siècles. Des arguments solides, au contraire, l'appuient, comme l'impressionnante uniformité du répertoire de la messe (non celui de l'office, beaucoup plus fluctuant), par delà les différentes familles manuscrites qui le véhiculent et dont les témoins deviennent légion dès le $\mathrm{XI}^{\mathrm{e}}$ siècle. Les premiers chercheurs qui se sont penchés sérieusement sur ces notations ont en effet été fort surpris de constater que les dissemblances de celles-ci étaient tout extérieures et masquaient une indiscutable convergence quant à la structure rythmique et à la courbe de la mélodie ; différences donc surtout graphiques, mais non point musicales. On peut s'en convaincre, très aisément, en comparant les trois "leçons» superposées du Graduale Triplex, qui proviennent de milieux fort éloignés : notations complémentaires comme on l'a vu, et se référant à un donné musical commun, indépendant en soi des signes qui en fixent la trace écrite. Même observation pour les notations sur portée qui ne s'écartent entre elles, le plus souvent, que dans des détails. Mais ces détails sont révélateurs des limites entre lesquelles se manifeste la variabilité de la tradition orale: variabilité pas plus arbitraire que celle qui commande les mutations phonétiques des mots parlés dont la linguistique a établi les lois générales. Et tout comme l'évolution de la prononciation et de l'accentuation latines se reflète au travers des inscriptions gravées dans la pierre jusqu'à la fin de l'Antiquité, ainsi un millénaire plus tard la tradition écrite du répertoire grégorien livre-t-elle, au gré d'un examen comparatif effectué sur une vaste échelle, maintes indications précieuses sur la pratique vivante de ce répertoire. Tel neume comprenant trois notes dans tel manuscrit, par exemple, se verra réduit à deux dans tel autre; mais l'élision n'affectera point n'importe laquelle des trois notes: ce sera toujours la même dans chacune des leçons de ce même passage, et toujours la même aussi dans d'autres spécimens du même neume. D'où l'on déduira avec une entière certitude que la note élidée avait un «poids» mélodique moindre que celui des deux autres - fût-ce en l'absence de toute différenciation graphique - et qu'elle s'exécutait par conséquent, quand elle n'était pas élidée, d'une manière plus légère que celles-ci ${ }^{14}$.

Quelles conséquences tirer de tout cela quant à la nature, à l'ancienneté et aux origines de la tradition grégorienne? Nous serions enclin pour notre part à voir dans la fixité «monolithique» du graduel un indice non négligeable de son âge vénérable et, partant, un gage de la fidélité de la tradition orale. Il faut faire ici appel au simple bon sens: l'unanimité substantielle de la tradition écrite des chants de la messe, derrière la diversité apparente des systèmes de notation, serait-elle crédible si cette tradition était contemporaine ou de peu antérieure aux premiers manuscrits notés où nous la trouvons, et si elle n'émanait point d'une autorité centrale capable de l'imposer à toute la chrétienté latine sous un aspect définitif et ne varietur, autorité qui ne saurait 
être que Rome ? Seul, semble-t-il, le siège pontifical était à même de susciter dans son entourage immédiat, par la concentration unique de forces actives réunies sous son obédience, cet immense travail de collation, de révision et d'achèvement qu'aura été la fixation du graduel grégorien, et ensuite de le répandre en sa pureté, sans déformations locales, dans la quasi-totalité des centres de la latinité chrétienne, ce à quoi la politique unificatrice des souverains carolingiens contribuera, comme on sait, dans une large mesure. On ne conçoit guère d'autre lieu que la Ville éternelle où situer le pôle de création et de diffusion d'une tradition orale conservée identique à elle-même au bout de deux ou trois siècles. L'histoire témoigne du prestige extraordinaire dont jouissait cette cité dans tout le monde chrétien dès le VII ${ }^{\mathrm{e}}$ siècle au moins. Une admiration universelle allait notamment à la somptueuse liturgie papale, que les Ordines romani nous font connaitre en ses moindres détails et dont la partie chantée était dévolue à la Schola cantorum, groupe réduit de garçons et de chantres adultes placé sous la haute main de l'«archichantre»: ce personnage considérable était aussi le supérieur des monastères rattachés à la basilique Saint-Pierre. On peut alors supposer que les mélodies récemment codifiées se sont transmises soit à Rome même à des chantres du dehors venus s'y former, soit ailleurs par des chantres romains affectés à cette mission de diffusion: le fait est attesté de manière certaine pour Rouen ${ }^{15}$, et on peut supposer qu'il en alla de même à Metz où l'évêque Chrodegang se fit le champion de la "romanisation» instaurée par Pépin le Bref vers 755. Quant au répertoire marginal qualifié de "vieux-romain", on devra admettre qu'il a coexisté à Rome (puisque les manuscrits qui le conservent s'y localisent tous) avec le répertoire "grégorien": plusieurs liturgistes envisagent comme possible cette coexistence de deux traditions distinctes, chacune appartenant alors à un milieu spécifique.

\section{Les traces écrites de l'oralité}

Laissons de côté ces épineux problèmes historiques, sur lesquels nous n'avons pu donner que de trop rapides aperçus, et abordons - trop brièvement, là aussi - le répertoire lui-même dans sa nature intrinsèque. Son rattachement au monde de l'oralité s'y décèle à un trait essentiel que l'on peut considérer comme l'un des caractères les plus significatifs de la composition grégorienne: nous voulons parler du formulisme, c'est-à-dire d'une technique compositionnelle fondée sur l'emploi d'un éventail de «formules », dessins et motifs musicaux servant d'éléments premiers à la création mélodique. On rejoint alors la notion générale du maqam (Idelsohn 1923; 1929: 24) bien connue des ethnomusicologues et qui équivaut à ce que Jacques Chailley (1960: 5-6) appelle le «mode formulaire», à savoir la complémentarité d'une échelle et d'un fonds mélodique où elle "s'incarne" (c'est aussi à peu près ce que désignent des termes comme l'allemand Tonweise ou Musikweise, et peutêtre le nomos de l'Antiquité grecque). Cette notion est illustrée en particulier par le chant synagogal dont découlent, au moins partiellement, tous les répertoires liturgiques chrétiens et qui s'est préservé, intact en ses principes, jusqu'à 
l'heure présente dans les diverses communautés de la «diaspora». Chaque livre - ou groupe de livres - biblique possède son "mode", c'est-à-dire son éventail de formules que le lecteur-chantre applique selon des règles strictes à la "cantillation» du texte sacré, lequel contient en regard des mots les signes conventionnels (taamim) correspondant à l'emplacement et à la nature des formules. De la liturgie synagogale la technique formulaire a passé dans les différents chants sacrés du christianisme oriental et occidental et notamment le chant grégorien, qui garde ainsi sous un visage en quelque sorte «pétrifié» par la tradition écrite cette pratique très représentative de l'oralité. D'un point de vue général, en effet, le formulisme apparaît comme une caractéristique fondamentale de tout enseignement oral transmis en milieu traditionnel, comme l'a montré l'étude anthropologique du phénomène de mémorisation entreprise par Marcel Jousse (voir Kovalevsky 1970). Sur le plan musical, il se réfère à une mémoire non sclérosée par l'envahissement de l'écrit et peuplée d'une multitude d' "images sonores », constitutives d'un héritage artistique qui ne vit que dans et par cet enracinement dans une mémoire vivante.

La technique formulaire affecte dans une proportion et sous des aspects variables les diverses formes grégoriennes. Celles de la pure cantillation, tout d'abord, avec les ponctuations mélodiques des lectures et oraisons ainsi que de la psalmodie (indiquées dans les lectionnaires médiévaux par des signes dont dérivent ceux que nous connaissons, à valeur devenue seulement grammaticale). Dans les couches archaïques des répons-graduels, traits et alléluias, ensuite, les formules prennent l'apparence plus élaborée de mélismes parfois assez amples, communs à plusieurs chants de même forme et de même mode, généralement en un endroit similaire (intonation, cadence, etc.) : ce sont les «mélismes migrateurs", en allemand wandernde Melismen. Dans la "composition libre" des antiennes d'introït, d'offertoire et de communion, enfin (et l'on pourrait leur associer, pour leur plus grande part, les antiennes de l'office), la présence des formules se déploie à deux niveaux et selon une souplesse telle que chaque mélodie fait corps avec le texte qu'elle commente: au niveau extérieur de l'ornementation, ce sont les brefs groupes de broderie, de passage ou autres qui soulignent et élargissent le profil accentuel du mot en lui-même et par rapport à la phrase; au niveau plus caché des schémas mélodiques sous-jacents, d'autre part, ce sont des courbes élémentaires articulées sur l'échelle pentatonique et reliant entre eux les degrés structuraux du mode (par exemple: $f a-s o l-l a-s o l-f a-r e ́$ en authente de ré ou plagal de $f a)^{16}$.

Une telle technique échappe à la mentalité rationaliste de l'Occident moderne, trop encline à penser en notes - «matière musicale» aussi peu significative en soi que les lettres de l'alphabet non groupées en mot - , alors que la mentalité traditionnelle de l'oralité n'envisage guère d'unités inférieures à la formule qui demeure de la "musique vivante». L'étude de la monodie grégorienne sera alors une voie précieuse pour nous ouvrir à un sentiment musical plus intuitif que rationnel, plus vécu en profondeur qu'intellectuellement réfléchi, plus affiné en qualité que mesuré en quantité. La reconstitution par la pensée de cette mentalité archaïque mais nullement «primitive» 
pourra seule nous faire comprendre d'autres faits, telle la parenté existant entre le grégorien et les «dialectes» parallèles du chant liturgique latin, vieuxromain, ambrosien (ou milanais) notamment. Souvent les mêmes chants figurent dans deux traditions voisines, sans qu'il y ait pratiquement aucune similitude textuelle : ici et là la mélodie suit le même parcours, s'attarde sur les mêmes intervalles, recourt à des formules similaires mais non identiques, avec parfois une divergence plus marquée de part et d'autre. Les deux traditions offrent des "cristallisations " corrélatives, unies entre elles par l'esprit et non par la lettre, d'un substrat musical mouvant et insaisissable, sorte d'archétype ou de matrice primordiale: source commune à laquelle toutes deux s'abreuvent et que notre analyse intellectualiste se révèle embarrassée à circonscrire parce qu'elle n'y retrouve guère ses catégories trop lucidement et rationnellement délimitées.

Disons quelques mots, pour conclure, de la tradition du chant grégorien aujourd'hui. Tradition qui, par la force des choses, n'est plus qu'écrite depuis de nombreux siècles, et dont la décadence a tragiquement révélé l'inaptitude de l'écriture à préserver valablement un héritage culturel et spirituel lorsqu'elle n'est plus doublée et «animée » par une tradition orale vivace. La «restauration grégorienne» de Dom Guéranger, vers 1850, n'aura pas été autre chose qu'un effort héroïque pour revivifier cette tradition, pour en retrouver la chair et le sang par delà l'écorce desséchée des documents écrits ${ }^{17}$. $Y$ est-on effectivement parvenu, et une semblable démarche a-t-elle même des chances d'aboutir ? Beaucoup estiment maintenant - non sans raison - que le grégorien issu de la "restauration" (et auquel restent fidèles, bon an mal an, la quasi-totalité des scholae actuelles) était perçu et recréé à travers la sensibilité musicale du XIX ${ }^{\mathrm{e}}$ siècle ; on peut en effet estimer que la douceur quelque peu lénifiante inséparable du «style grégorien » tel qu'on le conçoit ordinairement, s'éloigne passablement de la manière de chanter qui a dû être celle du Haut Moyen Age, manière qui nous paraîtrait probablement «orientale» ou " exotique» si, par miracle, il nous était donné de pouvoir la connaître de auditu. Il est alors tentant et peut-être prometteur de confronter la tradition écrite du chant grégorien avec la tradition orale, non interrompue jusqu'à l'heure présente, d'autres répertoires, pour s'aidant de ceux-ci, espérer retrouver quelque chose de l'esprit vivant du chant sacré latin traditionnel derrière la lettre morte des anciens manuscrits. Cette orientation constitue la phase la plus récente et la plus nouvelle de la musicologie grégorienne: on songe à la collaboration féconde ${ }^{18}$ du grégorianiste Marcel Pérès, chef de l'ensemble vocal "Organum», et de Lycourgos Angelopoulos, directeur du chœur byzantin d'Athènes; ou aux exécutions solistiques remarquables, nourries aux sources des chants folkloriques et traditionnels, de Dominique Vellard, professeur de chant médiéval à la Schola Cantorum de Bâle ${ }^{19}$. Quoi qu'on pense de ces tentatives, dont personne en tout cas ne saurait nier l'intérêt, on peut regarder comme un fait incontestable et de la plus haute importance que, sous quelque aspect qu'on l'aborde - en vue de sa juste exécution ou de son adéquate compréhension -, le chant grégorien ne sera jamais appréhendé en sa pleine et totale authenticité que dans la mesure où 
on le replacera dans l'éclairage de la tradition orale qui a régi autant sa gestation première que son magnifique épanouissement, celui de l'un de nos trésors musicaux et culturels les plus vénérables et les plus riches spirituellement.

\section{Notes}

1 Ces deux manuscrits ont été édités en fac-similé par Solesmes dans la collection de la Paléographie musicale: tomes X (Laon 239) et II, $2^{\mathrm{e}}$ série (Saint-Gall 359).

2 Paléographie musicale, tome $\mathrm{I}, 2^{\mathrm{e}}$ série.

3 Voir également Suñol (1935: 31$)$.

${ }^{4}$ On conserve au total six graduels sans notation, publiés dans Hesbert (1935).

5 Cf. Dom André Mocquereau, dans Paléographie musicale, tome I, Solesmes, 1889, p. 106. Rappelons cependant qu'il existe une notation neumatique archaïque, dite "paléofranque", qui serait apparue vers 830 dans le nord de la France et dont dérivent les deux notations bretonne et lorraine (Huglo 1976, II : 659).

6 Comme argument a contrario on cite la phrase d'Isidore de Séville (570-636): Nisi ab homine memoria teneantur, soni pereunt, quia scribi non possunt («si les sons ne sont pas retenus par l'homme dans sa mémoire, ils périssent, car on ne peut pas les retenir"; Etymologies, 15, 2) (Chailley $1969: 75$ ).

7 Et même trois, si l'on tient compte de la notation «dasiane» employée dans le traité Musica Enchiriadis d'Ogier de Laon (début du IX ${ }^{\mathrm{e}}$ siècle), et dont Guy d'Arezzo reprendra le principe de la portée et des clés.

${ }^{8}$ Ce manuscrit provient en réalité de Saint-Bénigne de Dijon, et a été édité par Solesmes (Paléographie musicale, tome VIII). Les chants y figurent en deux notations superposées, alphabétique et neumatique, combinaison préconisée par Hucbald de Saint-Amand un siècle plus tôt (cf. Gerbert, I: 117).

9 Dans ses Regulae musicae rythmicae (Gerbert, II : 30), Guy d'Arezzo semble suggérer que la préférence accordée à la notation neumatique par rapport à son homologue alphabétique serait due à un gain de temps. Gain peut-être pour le copiste, mais non assurément pour l'apprentissage du répertoire, comme on le verra! Remarquons à ce propos que Guy semble réellement obsédé par la préoccupation de tout ramener à des mobiles pragmatiques et utilitaires : ainsi par exemple les syllabes de solmisation ( $u t, r e ́, m i$, etc.) dont il tire sa méthode bien connue, base du moderne solfège, étaient à l'origine chargées d'un symbolisme ésotérique dont il n'a cure, comme nous l'avons montré ailleurs (Viret 1986: chap. VI ; 1988 : chap. IV; Viret et Chailley 1988).

${ }^{10}$ Epistola Michaeli monacho (Gerbert, II : 43).

${ }^{11}$ Smits van Waesberghe (1969: 25); voir également Viret (1985).

${ }^{12}$ Graduale Triplex, Solesmes, 1979 dont le texte mélodique est celui du Graduale Romanum de 1974, selon la nouvelle liturgie de Paul VI.

${ }^{13}$ Voir Wildhaber (1985) et Hakkennes (1985) qui ont tous deux présenté des tentatives de cet ordre. Nous avons nous-même proposé une notation synthétique (Viret 1986).

${ }^{14}$ L'exemple cité est celui du «torculus spécial» étudié par Dom Eugène Cardine (1970: 29-34).

${ }^{15}$ Sur la foi d'une lettre du pape Paul I ${ }^{\mathrm{er}}$ à Pépin le Bref dont le frère était l'évêque Rémédius de Rouen, lequel put accueillir dans son diocèse le sous-directeur de la Schola cantorum romaine avant d'envoyer à Rome ses propres chantres (cf. Migne, Patrologie latine, LXXXIX, col. 187).

${ }^{16}$ Cet aspect important de la composition grégorienne a été étudié par nous-même (Viret 1986, 1988). 
${ }^{17}$ C'est encore cette même démarche, très exactement, qui inspire la sémiologie mise en honneur vers 1950 par Dom Eugène Cardine et qui a polarisé depuis lors l'essentiel de la recherche et de l'enseignement grégoriens, en France tout au moins. La réelle valeur scientifique des travaux réalisés dans cette voie ne doit cependant pas faire oublier les limites de celle-ci : l'étude, si minutieuse soit-elle, des signes graphiques demeurera toujours à la surface ou en marge du fait musical conçu globalement.

${ }^{18}$ Collaboration qui porte, il est vrai, surtout sur le répertoire dit vieux-romain, d'un caractère incontestablement plus oriental que le grégorien. Mais l'orientalisme de ce dernier n'en est pas moins indiscutable, en considérant par exemple les neumes ornementaux (quilisma, oriscus, tristropha, pressus, trigon, etc.) qui, d'après divers auteurs contemporains, correspondent à de subtils effets de voix, dont la tradition se serait perdue de très bonne heure mais qui sont peut-être analogues à ceux que l'on observe dans les chants traditionnels actuels. Ces neumes impliquent probablement l'emploi des microintervalles, indiqués selon certains par quelques particularités de la notation alphabétique dans le manuscrit de Saint-Bénigne de Dijon (PM, tome VIII). Voir le disque Chants de l'Eglise de Rome des $V I I^{e}$ et $V I I I^{e}$ siècles, période byzantine, par l'ensemble vocal "Organum» dirigé par Marcel Pérès, avec Lycourgos Angelopoulos (Harmonia Mundi/ France, HMC 901218, 1986). Nous avons appris que Marcel Pérès orientait actuellement ses recherches vers la tradition syriaque, dans un même esprit comparatif.

${ }^{19}$ Nous en jugeons d'après le concert radiodiffusé donné le 31 mars 1987 dans les locaux de l'ORTF, Paris, par Dominique Vellard et Emmanuel Bonnardot (le répertoire des chantres $\mathrm{du} \mathrm{X} \mathrm{X}^{\mathrm{e}}$ au XIII ${ }^{\mathrm{e}}$ siècle).

\section{Bibliographie}

CARDINE Dom Eugène

1970 Sémiologie grégorienne. Solesmes.

CHAILLEY Jacques

1960 L'imbroglio des modes. Paris : Leduc.

1969 Histoire musicale du Moyen Age. Paris: PUF ( $2^{\mathrm{e}}$ éd.)

\section{GERBERT Martin}

1784 Scriptores ecclesiastici de musica. Saint-Blaise (3 vol.).

HAKKENNES Chris F.J.

1985 «Présentation du Graduale Lagal». Actes du Congrès international de chant grégorien. Paris, p. 65-81 (multigraphié).

HESBERT Dom René-Jean

1935 Antiphonale Missarum Sextuplex. Bruxelles.

HUGLO Michel

1976 «Neume». In : dictionnaire Science de la musique. Paris : Bordas, tome II : 658-659.

IDELSOHN Abraham Z.

1913-14 «Die Maqamen der arabischen Musik». In: Sammelbände der Internationalen Musikgesellschaft XV : 11-63 (repris dans Hebräisch-Orientalischer Melodienschatz, IV, Leipzig, 1923, p. 62-112).

1929 Jewish music in its historical development. New York. 


\section{KOVALEVSKY Maxime}

1970 «Le formulisme dans la musique liturgique chrétienne». In: Encyclopédie des musiques sacrées, III : 479-87. Paris : Labergerie.

SMITS VAN WAESBERGHE J.

1957 «Etat actuel des recherches scientifiques dans le domaine du chant grégorien». Actes du Troisième Congrès international de musique sacrée. Paris : Ed. du Congrès, p. 206-217.

1969 Lehre und Theorie der Musik im Mittelalter. Leipzig: VEB Deutscher Verlag für Musik (Musikgeschichte in Bildern, III/3).

SUÑOL Dom Grégoire

1935 Introduction à la paléographie musicale grégorienne. Tournai : Desclée.

VIRET Jacques

1985 «L'enseignement musical au Moyen Age». Chant choral (Lyon) 45(1) : 10-23.

1986 Le chant grégorien. Lausanne : L'Age d'Homme.

1988 La modalité grégorienne. Lyon: A Cœur Joie.

VIRET Jacques et CHAILLEY Jacques

1988 «Le symbolisme de la gamme». La Revue musicale.

WILDHABER Dom Bruno-Marie

1985 «Le chant de Citeaux entre saint Bernard et saint Grégoire». Actes du Congrès international de chant grégorien. Paris. p. 137-151 (multigraphié). 